\title{
Reach-SDP: Reachability Analysis of Closed-Loop Systems with Neural Network Controllers via Semidefinite Programming
}

\author{
Haimin Hu, Mahyar Fazlyab, Manfred Morari, and George J. Pappas*
}

\begin{abstract}
There has been an increasing interest in using neural networks in closed-loop control systems to improve performance and reduce computational costs for on-line implementation. However, providing safety and stability guarantees for these systems is challenging due to the nonlinear and compositional structure of neural networks. In this paper, we propose a novel forward reachability analysis method for the safety verification of linear time-varying systems with neural networks in feedback interconnection. Our technical approach relies on abstracting the nonlinear activation functions by quadratic constraints, which leads to an outer-approximation of forward reachable sets of the closed-loop system. We show that we can compute these approximate reachable sets using semidefinite programming. We illustrate our method in a quadrotor example, in which we first approximate a nonlinear model predictive controller via a deep neural network and then apply our analysis tool to certify finite-time reachability and constraint satisfaction of the closed-loop system.
\end{abstract}

\section{Introduction}

Deep neural networks (DNN) have seen renewed interest in recent years due to the proliferation of data and access to more computational power. In autonomous systems, DNNs are either used as feedback controllers [17, 35], motion planners [31], perception modules, or end-to-end controllers [5, 28. Despite their high performance, DNN-driven autonomous systems lack formal safety and stability guarantees. Indeed, recent studies show that DNNs can be vulnerable to small perturbations or adversarial attacks [23, 29]. This issue is more pronounced in closed-loop systems, as a small perturbation in the loop can dramatically change the behavior of the closed-loop system over time. Therefore, it is of utmost importance to develop tools for verification of DNN-driven control systems. The goal of this paper is to develop a methodology, based on semidefinite programming, for safety verification and reachability analysis of linear dynamical systems in feedback interconnection with DNNs.

Safety verification or reachability analysis aims to show that starting from a set of initial conditions, a dynamical system cannot evolve to an unsafe region in the state space. Methods for reachability analysis can be categorized into exact (complete) or approximate (incomplete), which compute the reachable sets exactly and approximately, respectively. Verification of dynamical systems has been extensively studied in the past [2, 3, 24, 30, 33. More recently, the problem of

\footnotetext{
${ }^{*}$ Work supported by the NSF under grants DARPA Assured Autonomy and NSF CPS 1837210. The authors are with the Department of Electrical and Systems Engineering, University of Pennsylvania. Email: \{haiminhu,mahyarfa,morari,pappasg\}@seas.upenn.edu.
} 
output range analysis of neural networks has been addressed in $19,10,12,13,19,21,22,26,32$, mainly motivated by robustness analysis of DNNs against adversarial attacks [23]. Compared to these bodies of work, verification of closed-loop systems with neural network controllers has been less explored [8, 18, 20]. In [20], a method for verification of sigmoid-based neural networks in feedback with a hybrid system is proposed, in which the neural network is transformed into a hybrid system and then a standard verification tool for hybrid systems is invoked. In [18, a new reachability analysis approach based on Bernstein polynomials is proposed that can verify DNNcontrolled systems with Lipschitz continuous activation functions. Dutta et al. [8] use a flow pipe construction scheme to over approximate the reachable sets. A piecewise polynomial model is used to provide an approximation of the input-output mapping of the controller and an error bound on the approximation. This approach, however, is only applicable to Rectified Linear Unit (ReLU) activation functions.

Contributions. In this paper, we propose a semidefinite program (SDP) for reachability analysis of linear time-varying dynamical systems in feedback interconnection with neural network controllers equipped with a projection operator, which projects the output of the neural network (the control action) onto a specified set of control inputs. Our technical approach relies on abstracting the nonlinear activation functions as well as the projection operator by quadratic constraints [12], which leads to an outer-approximation of forward reachable sets of the closed-loop system. We show that we can compute these approximate reachable sets using semidefinite programming. Our approach can be used to analyze control policies learned by neural networks in, for example, model predictive control (MPC) [7] and constrained reinforcement learning [34]. To the best of our knowledge, our result is the first convex-optimization-based method for reachability analysis of closed-loop systems with neural networks in the loop. We illustrate the utility of our approach in two numerical examples, in which we certify finite-time reachability and constraint satisfaction of a double integrator and a quadrotor.

\subsection{Notation and Preliminaries}

We denote the set of real numbers by $\mathbb{R}$, the set of real $n$-dimensional vectors by $\mathbb{R}^{n}$, the set of $m \times n$-dimensional matrices by $\mathbb{R}^{m \times n}$, and the $n$-dimensional identity matrix by $I_{n}$. We denote by $\mathbb{S}^{n}, \mathbb{S}_{+}^{n}$, and $\mathbb{S}_{++}^{n}$ the sets of $n$-by- $n$ symmetric, positive semidefinite, and positive definite matrices, respectively. For $A \in \mathbb{R}^{m \times n}$, the inequality $A \geq 0$ means all entries of $A$ are non-negative. For $A \in \mathbb{S}^{n}$, the inequality $A \succeq 0$ means $A$ is positive semidefinite.

Definition 1 (Sector-bounded nonlinearity). A nonlinear function $\varphi: \mathbb{R} \rightarrow \mathbb{R}$ is sector-bounded on $[\alpha, \beta]$, where $0 \leq \alpha \leq \beta$, if the following inequality holds for all $x \in \mathbb{R}$,

$$
\alpha \leq \frac{\varphi(x)}{x} \leq \beta
$$

which can be equivalently expressed as the quadratic inequality

$$
\left[\begin{array}{c}
x \\
\varphi(x)
\end{array}\right]^{\top}\left[\begin{array}{cc}
-2 \alpha \beta & \alpha+\beta \\
\alpha+\beta & -2
\end{array}\right]\left[\begin{array}{c}
x \\
\varphi(x)
\end{array}\right] \geq 0 .
$$

Definition 2 (Slope-restricted nonlinearity). A nonlinear function $\varphi: \mathbb{R} \rightarrow \mathbb{R}$ is slope-restricted on $[\alpha, \beta]$, where $0 \leq \alpha \leq \beta$, if for any pairs of $(x, \varphi(x))$ and $\left(x^{\star}, \varphi\left(x^{\star}\right)\right)$,

$$
\alpha \leq \frac{\varphi(x)-\varphi\left(x^{\star}\right)}{x-x^{\star}} \leq \beta,
$$


which can be equivalently expressed as

$$
\left[\begin{array}{c}
x-x^{\star} \\
\varphi(x)-\varphi\left(x^{\star}\right)
\end{array}\right]^{\top}\left[\begin{array}{cc}
-2 \alpha \beta & \alpha+\beta \\
\alpha+\beta & -2
\end{array}\right]\left[\begin{array}{c}
x-x^{\star} \\
\varphi(x)-\varphi\left(x^{\star}\right)
\end{array}\right] \geq 0 .
$$

\section{Problem Formulation}

\subsection{Neural Network Control System}

We consider a discrete-time linear time-varying system

$$
P: x_{t+1}=A_{t} x_{t}+B_{t} u_{t}+c_{t},
$$

where $x_{t} \in \mathbb{R}^{n_{x}}, u_{t} \in \mathbb{R}^{n_{u}}$ are the state and control vectors, and $c_{t} \in \mathbb{R}^{n_{x}}$ is an exogenous input. We assume that the system (5) is subject to input constraints,

$$
u_{t} \in \mathcal{U}_{t}, t=0,1, \cdots .
$$

which represent, for example, actuator limits that are naturally satisfied or hard constraints that must be satisfied by a control design specification. A specialization that we consider in this paper is input box constraint $\mathcal{U}_{t}=\left\{u_{t} \mid \underline{u}_{t} \leq u_{t} \leq \bar{u}_{t}\right\}$. Furthermore, we assume a state-feedback controller $\pi\left(x_{t}\right): \mathbb{R}^{n_{x}} \rightarrow \mathbb{R}^{n_{u}}$ parameterized by a multi-layer feed-forward fully-connected neural network. The map $x \mapsto \pi(x)$ is described by the following equations,

$$
\begin{aligned}
x^{0} & =x \\
x^{k+1} & =\phi\left(W^{k} x^{k}+b^{k}\right) \quad k=0, \cdots, \ell-1 \\
\pi(x) & =W^{\ell} x^{\ell}+b^{\ell},
\end{aligned}
$$

where $W^{k} \in \mathbb{R}^{n_{k+1} \times n_{k}}, b^{k} \in \mathbb{R}^{n_{k+1}}$ are the weight matrix and bias vector of the $(k+1)$-th layer. The nonlinear activation function $\phi(\cdot)$ is applied component-wise to the pre-activation vectors, i.e.,

$$
\phi(x):=\left[\varphi\left(x_{1}\right) \cdots \varphi\left(x_{d}\right)\right]^{\top}, x \in \mathbb{R}^{d},
$$

where $\varphi: \mathbb{R} \rightarrow \mathbb{R}$ is the activation function of each individual neuron. Common choices include ReLU, sigmoid, tanh, leaky ReLU, etc. In this paper, we consider ReLU activation functions in our technical derivations but we can address other activation functions following the framework of [12]. To ensure that output of neural network respects the input constraint, we consider a projection operator in the loop and define the control input as

$$
u_{t}=\operatorname{Proj}_{\mathcal{U}_{t}}\left(\pi\left(x_{t}\right)\right)
$$

We denote the closed-loop system with dynamics (5) and the projected neural network control policy (9) by

$$
x_{t+1}=f_{\pi}\left(x_{t}\right),
$$

which is a non-smooth nonlinear system because of the presence of the nonlinear activation functions in the neural network and the projection operator.

Remark 1. In this paper, we only consider box constraints for the input and leave more sophisticated constraints such as polytopes to future work. These constraints are useful in, for example, $M P C$ design problems. In [17] a robust MPC controller is approximated by a neural network equipped with a projection operator to ensure satisfaction of polytopic input constraints. 
For the closed-loop system (10) subject to the input constraint in $(6)$, we denote by $\mathcal{R}_{t}\left(\mathcal{X}_{0}\right)$ the forward reachable set at time $t$ from a given set of initial conditions $\mathcal{X}_{0} \subseteq \mathbb{R}^{n_{x}}$, which is defined by the recursion

$$
\mathcal{R}_{t+1}\left(\mathcal{X}_{0}\right):=f_{\pi}\left(\mathcal{R}_{t}\left(\mathcal{X}_{0}\right)\right), \mathcal{R}_{0}\left(\mathcal{X}_{0}\right)=\mathcal{X}_{0}
$$

as illustrated in Figure 1.



Figure 1: An illustration of closed-loop reachability with the initial set $\mathcal{X}_{0}$, the $t$-step forward reachable set $\mathcal{R}_{t}\left(\mathcal{X}_{0}\right)$, and its over-approximation $\overline{\mathcal{R}}_{t}\left(\mathcal{X}_{0}\right)$ shown in magenta.

\subsection{Finite-Time Reach-Avoid Verification Problem}

In this paper, we are interested in verifying the finite-time reach-avoid properties of the closed-loop system (10). More specifically, given a goal set $\mathcal{G} \subseteq \mathbb{R}^{n_{x}}$ and a sequence of avoid sets $\mathcal{A}_{t} \subseteq \mathbb{R}^{n_{x}}$, we would like to test if all initial states $x_{0} \in \mathcal{X}_{0}$ of the closed-loop system 110 can reach $\mathcal{G}$ in a finite time horizon $N \geq 0$, while avoiding $\mathcal{A}_{t}$ for all $t=0, \cdots, N$. This is equivalent to test if,

$$
\begin{aligned}
& \mathcal{R}_{N}\left(\mathcal{X}_{0}\right) \subseteq \mathcal{G} \\
& \mathcal{R}_{t}\left(\mathcal{X}_{0}\right) \cap \mathcal{A}_{t}=\emptyset, \forall t=0, \cdots, N
\end{aligned}
$$

holds true for (10). There exist efficient methods [4] and software implementations [16] for testing set inclusion (12a) and set intersection 12b. However, computing exact reachable sets for the nonlinear closed-loop system (10) is, in general, computationally intractable. Therefore, we resort to finding outer-approximations of the closed-loop reachable sets, $\overline{\mathcal{R}}_{t}\left(\mathcal{X}_{0}\right) \supseteq \mathcal{R}_{t}\left(\mathcal{X}_{0}\right)$, and use them to test if,

$$
\begin{aligned}
& \overline{\mathcal{R}}_{N}\left(\mathcal{X}_{0}\right) \subseteq \mathcal{G} \\
& \overline{\mathcal{R}}_{t}\left(\mathcal{X}_{0}\right) \cap \mathcal{A}_{t}=\emptyset, \quad \forall t=0, \cdots, N
\end{aligned}
$$

is true. Note that (13) are sufficient conditions for (12). To obtain meaningful certificates, we want the approximations $\mathcal{R}_{t}\left(\mathcal{X}_{0}\right)$ to be as tight as possible. Thus our goal is to compute the tightest outer-approximations of the $t$-step reachable sets. This can be addressed, for example, by solving the following optimization problem,

$$
\begin{aligned}
\text { minimize } & \text { Volume }\left(\overline{\mathcal{R}}_{t}\left(\mathcal{X}_{0}\right)\right) \\
\text { subject to } & \mathcal{R}_{t}\left(\mathcal{X}_{0}\right) \subseteq \overline{\mathcal{R}}_{t}\left(\mathcal{X}_{0}\right) .
\end{aligned}
$$

The solution to the above problem is the minimum-volume outer-approximation of the $t$-step reachable set of the closed-loop system (10). In the following sections, we will derive a convex relaxation to the optimization problem (14). 


\section{Problem Abstraction via Quadratic Constraints}

This section focuses on estimating the one-step forward reachable set $\overline{\mathcal{R}}\left(\mathcal{X}_{0}\right)$. The main idea is to replace the original closed-loop system $f_{\pi}$ with an abstracted system $\tilde{f}_{\pi}$ in the sense that $\tilde{f}_{\pi}$ overapproximates the output of the original system for any given initial set $\mathcal{X}_{0}$, i.e. $\mathcal{R}\left(\mathcal{X}_{0}\right)=f_{\pi}\left(\mathcal{X}_{0}\right) \subseteq$ $\tilde{f}_{\pi}\left(\mathcal{X}_{0}\right)=\overline{\mathcal{R}}\left(\mathcal{X}_{0}\right)$. Based on this abstracted system, we can then compute $\overline{\mathcal{R}}\left(\mathcal{X}_{0}\right)$ via semidefinite programming (SDP). In the following, we will develop such an abstraction using the framework of Quadratic Constraints (QCs).

\subsection{Initial Set}

We begin with a formal definition of QCs for sets [12].

Definition 3 (Quadratic Constraints). Let $\mathcal{X} \subset \mathbb{R}^{d}$ be a nonempty set and $\mathcal{Q} \subset \mathbb{S}^{d+1}$ be the set of all symmetric, but possibly indefinite matrices $Q$ such that the inequality

$$
\left[\begin{array}{l}
x \\
1
\end{array}\right]^{\top} Q\left[\begin{array}{l}
x \\
1
\end{array}\right] \geq 0
$$

holds for all $x \in \mathcal{X}$. Then we say $\mathcal{X}$ satisfies the $Q C$ defined by $\mathcal{Q}$. The vector $\left[x^{\top} 1\right]^{\top}$ is called the basis of this $Q C$.

Here, for each fixed $Q$, the set of $x$ 's satisfying $(15)$ is a superset of $\mathcal{X}$. Indeed, we have that

$$
\mathcal{X} \subseteq \bigcap_{Q \in \mathcal{Q}}\left\{x \in \mathbb{R}^{d} \mid\left[\begin{array}{l}
x \\
1
\end{array}\right]^{\top} Q\left[\begin{array}{l}
x \\
1
\end{array}\right] \geq 0\right\} .
$$

In this paper, we mainly use polytopes and ellipsoids as the initial set $\mathcal{X}_{0}$. Nonetheless, as addressed by [12, other types of sets such as hyper-rectangles and zonotopes are also applicable in this setting.

Proposition 1 (QC for polytope). Suppose the initial set $\mathcal{X}_{0}$ is a polytope defined by $\mathcal{X}_{0}=$ $\left\{x \in \mathbb{R}^{n_{x}} \mid A x \leq b\right\}$. Then $\mathcal{X}_{0}$ satisfies the $Q C$ defined by

$$
\mathcal{P}=\left\{P \mid P=\left[\begin{array}{cc}
A^{\top} \Gamma A & -A^{\top} \Gamma b \\
-b^{\top} \Gamma A & b^{\top} \Gamma b
\end{array}\right], \Gamma \in \mathbb{S}^{m}, \Gamma \geq 0\right\},
$$

where $m$ is the dimension of $b$. The basis is $\left[\begin{array}{ll}x^{\top} & 1\end{array}\right]^{\top}$.

Proposition 2 (QC for ellipsoid). Suppose the initial set $\mathcal{X}_{0}$ is an ellipsoid defined by $\mathcal{X}_{0}=$ $\left\{x \in \mathbb{R}^{n_{x}} \mid\|A x+b\|_{2} \leq 1\right\}$, where $A \in \mathbb{S}^{n_{x}}$ and $b \in \mathbb{R}^{n_{x}}$. Then $\mathcal{X}_{0}$ satisfies the $Q C$ defined by

$$
\mathcal{P}=\left\{P \mid P=\mu\left[\begin{array}{cc}
-A^{\top} A & -A^{\top} b \\
-b^{\top} A & 1-b^{\top} b
\end{array}\right], \mu \geq 0\right\}
$$

with basis $\left[x^{\top} 1\right]^{\top}$.

To this end, we have over-approximated the initial set $\mathcal{X}_{0}$ and represented it as QCs. We will see in Section 4 that the matrix $P \in \mathcal{P}$ appears as a decision variable in the SDP. It provides an extra degree of freedom towards a less conservative estimation of the reachable set $\overline{\mathcal{R}}\left(\mathcal{X}_{0}\right)$. 


\subsection{Reachable Set}

In order to facilitate the relaxation of the volume-minimization problem in (14), we assume the candidate set $\overline{\mathcal{R}}\left(\mathcal{X}_{0}\right)$ that over-approximates $\mathcal{R}\left(\mathcal{X}_{0}\right)$ is represented by the intersection of finitely many quadratic inequalities:

$$
\overline{\mathcal{R}}\left(\mathcal{X}_{0}\right)=\bigcap_{i=1}^{m}\left\{x \in \mathbb{R}^{n_{x}} \mid\left[\begin{array}{l}
x \\
1
\end{array}\right]^{\top} S_{i}\left[\begin{array}{l}
x \\
1
\end{array}\right] \leq 0\right\},
$$

where matrices $S_{i} \in \mathbb{S}^{2 n_{x}+1}$ capture the shape and volume of the reachable set and will appear as decision variables in the SDP problem. Typically, (19) is able to describe polytopic and ellipsoidal sets, which we discuss in detail now.

\subsubsection{Polytopic reachable set}

If the reachable set is to be over-approximated by a polytope, i.e. $\overline{\mathcal{R}}\left(\mathcal{X}_{0}\right)=\left\{x \in \mathbb{R}^{n_{x}} \mid A f_{\pi}(x) \leq b\right\}$, then,

$$
S_{i}=\left[\begin{array}{cc}
0 & a_{i}^{\top} \\
a_{i} & -2 b_{i}
\end{array}\right]
$$

where $a_{i} \in \mathbb{R}^{1 \times n_{x}}$ is the $i$-th row of $A \in \mathbb{R}^{m \times n_{x}}$ and $b_{i} \in \mathbb{R}$ is the $i$-th entry of $b \in \mathbb{R}^{m}$. Here, we require the $A$ matrix, which determines the orientation of each facet of the polytope, to be given while we leave $b_{i}$ 's as decision variables in the SDP problem.

\subsubsection{Ellipsoidal reachable set}

If the reachable set is to be described by an ellipsoid, i.e. $\overline{\mathcal{R}}\left(\mathcal{X}_{0}\right)=\left\{x \in \mathbb{R}^{n_{x}} \mid\left\|A f_{\pi}(x)+b\right\|_{2} \leq 1\right\}$, then,

$$
S_{i}=S=\left[\begin{array}{cc}
A^{\top} A & A^{\top} b \\
b^{\top} A & b^{\top} b-1
\end{array}\right]
$$

where $A \in \mathbb{S}^{n_{x}}$ and $b \in \mathbb{R}^{n_{x}}$ are decision variables describing the center, orientation, and volume of the ellipsoid.

Remark 2. For polytopic reachable sets, if the facets $a_{i}$ are properly chosen, the resulting outerapproximations can be very tight, as we will see in Section 5.1. However, finding facets of a higher dimensional polytope can be prohibitively challenging. Ellipsoidal reachable sets scale better and therefore are more suitable in this case.

\subsection{ReLU Neural Networks with Projection}

In this subsection, we show how to abstract the nonlinear activation functions of the neural network by QCs. We will focus on the ReLU function throughout the paper. Other types of activation functions such as sigmoid and tanh can also be represented by QCs. See [12] for details. Recall that the input constraint sets are $\mathcal{U}_{t}=\left\{u_{t} \mid \underline{u}_{t} \leq u_{t} \leq \bar{u}_{t}\right\}$. Consider the following recursion,

$$
\begin{aligned}
x_{t}^{0} & =x_{t} \\
x_{t}^{k+1} & =\max \left(W^{k} x_{t}^{k}+b^{k}, 0\right) \quad k=0, \cdots, \ell-1 \\
x_{t}^{\ell+1} & =\max \left(W^{\ell} x_{t}^{\ell}+b^{\ell}-\underline{u}_{t}, 0\right)+\underline{u}_{t} \\
x_{t}^{\ell+2} & =-\max \left(\bar{u}_{t}-x_{t}^{\ell+1}, 0\right)+\bar{u}_{t} .
\end{aligned}
$$


Then it is not hard to show that $x_{t}^{\ell+2}=u_{t}=\operatorname{Proj}_{\mathcal{U}_{t}}\left(\pi\left(x_{t}\right)\right)$. In other words, we have embedded the projection operator $(9)$ as two additional layers into the neural network.

Now, we derive the QCs for the ReLU activation function, $\phi(x)=\max (0, x), x \in \mathbb{R}^{d}$, which is the nonlinearity used in the hidden layers of the projected neural network. Note that for $d=1$, this function is on the boundary of the sector $[0,1]$. More precisely, we can describe it by taking the intersection of three quadratic and/or affine constraints:

$$
y_{i}=\max \left(0, x_{i}\right) \Leftrightarrow y_{i} \geq x_{i}, y_{i} \geq 0, y_{i}^{2}=x_{i} y_{i},
$$

for all $i=1, \cdots, d$. In addition, the $\operatorname{ReLU} \phi(x)$ is also slope-restricted on $[0,1]$ with repeated nonlinearity. This allows us to write (4) with $\alpha=0$ and $\beta=1$,

$$
\left(y_{j}-y_{i}\right)^{2} \leq\left(y_{j}-y_{i}\right)\left(x_{j}-x_{i}\right), \forall i \neq j .
$$

By taking a weighted sum of the constraints (23) and (24), we obtain the single quadratic constraint,

$$
\begin{aligned}
& \sum_{i=1}^{d} \lambda_{i}\left(y_{i}^{2}-x_{i} y_{i}\right)+\nu_{i}\left(y_{i}-x_{i}\right)+\eta_{i} y_{i}- \\
& \sum_{i \neq j} \lambda_{i j}\left(\left(y_{j}-y_{i}\right)^{2}-\left(y_{j}-y_{i}\right)\left(x_{j}-x_{i}\right)\right) \geq 0,
\end{aligned}
$$

which holds for any multipliers $\left(\lambda_{i}, \nu_{i}, \eta_{i}, \lambda_{i j}\right) \in \mathbb{R} \times \mathbb{R}_{+}^{3}$ and $i, j \in\{1, \cdots, d\}$. The following lemma shows how to express the above constraint in a standard-form QC (15).

Lemma 1 (QC for ReLU function). The ReLU function $\phi(x)=\max (0, x): \mathbb{R}^{d} \rightarrow \mathbb{R}^{d}$ satisfies the $Q C$ defined by

$$
\mathcal{Q}=\left\{Q \mid Q=\left[\begin{array}{ccc}
0 & T & -\nu \\
T & -2 T & \nu+\eta \\
-\nu^{\top} & \nu^{\top}+\eta^{\top} & 0
\end{array}\right]\right\} .
$$

with basis $\left[x^{\top} \phi(x)^{\top} 1\right]^{\top}$. Here, $\eta, \nu \geq 0$ and $T \in \mathbb{S}_{+}^{d}$ is given by

$$
T=\sum_{i=1}^{d} \lambda_{i} e_{i} e_{i}^{\top}+\sum_{i=1}^{d-1} \sum_{j>i}^{d} \lambda_{i j}\left(e_{i}-e_{j}\right)\left(e_{i}-e_{j}\right)^{\top},
$$

where $\lambda_{i j} \geq 0$ and $e_{i} \in \mathbb{R}^{d}$ has 1 in the $i$-th entry and 0 everywhere else.

Proof. See [12].

As we will see in Section 4 , the $Q$ matrix in (26) will appear as a decision variable in the SDP problem. Note that there are many ways to refine $(26)$ to yield a tighter relaxation for a specific region in the state-space, such as using interval arithmetic [11, 12] or linear programming [22].

\section{Reach-SDP: Computing Forward Reachable Sets via Semidefi- nite Programming}

In this section, we propose Reach-SDP, an optimization-based approach that uses the QC abstraction developed in the previous section to estimate the reachable set of the closed-loop system (10). Specifically, the $N$-step reachable set is estimated using the following recursive computations,

$$
\overline{\mathcal{R}}_{t+1}\left(\mathcal{X}_{0}\right)=\text { Reach_SDP }\left(\overline{\mathcal{R}}_{t}\left(\mathcal{X}_{0}\right)\right),
$$

for $t=0, \cdots, N-1$. In the sequel, we discuss how to implement Reach_SDP in detail. 


\subsection{Change of Basis}

In the previous section, we have abstracted the initial set, the reachable set and the projected neural network with QCs or quadratic inequalities, each with a different basis vector. For Reach-SDP, we unify those quadratic terms with the same basis vector:

$$
\left[\mathbf{x}^{\top} 1\right]^{\top}:=\left[\begin{array}{lll}
x_{t}^{0^{\top}} & x_{t}^{1^{\top}} \cdots & x_{t}^{\ell+2}
\end{array}\right]^{\top} \in \mathbb{R}^{n_{x}+n_{n}+2 n_{u}+1},
$$

where $n_{n}=\sum_{k=1}^{\ell} n_{k}$ is the total number of neurons in the neural network. Then, the unified QC is in the form:

$$
\left[\begin{array}{c}
\mathbf{x} \\
1
\end{array}\right]^{\top} M\left[\begin{array}{c}
\mathbf{x} \\
1
\end{array}\right] \geq 0,
$$

where $M \in \mathbb{S}^{n_{x}+n_{n}+1}$. The following lemma shows how to change the basis of a QC by a congruence transformation.

Lemma 2. The $Q C$ defined by $\mathcal{Q}=\left\{Q \in \mathbb{S}^{d} \mid x_{b}^{\top} Q x_{b} \geq 0\right\}$ with basis $x_{b} \in \mathbb{R}^{d}$ is equivalent to the $Q C$ defined by $\mathcal{M}=\left\{M \in \mathbb{S}^{n} \mid \xi_{b}^{\top} M \xi_{b} \geq 0\right\}$ with basis $\xi_{b} \in \mathbb{R}^{n}$, where $M=E^{\top} Q E$ and $E \xi_{b}=x_{b}$. The matrix $E \in \mathbb{R}^{n \times d}$ is the change-of-basis matrix.

Proof. Substitute $E \xi_{b}=x_{b}$ into $x_{b}^{\top} Q x_{b} \geq 0$ and we have $x_{b}^{\top} E^{\top} Q E x_{b}=\xi_{b}^{\top} M \xi_{b} \geq 0$.

Proposition 3. The $Q C$ defined by $\mathcal{P}$ in $(17)$ or $(18)$, satisfied by the initial set $\mathcal{X}_{0}$, is equivalent to the $Q C$ defined by $\mathcal{M}_{\mathrm{in}}=\left\{M_{\mathrm{in}}(P) \in \mathbb{S}^{n_{x}+n_{n}+1} \mid M_{\mathrm{in}}(P)=E_{\mathrm{in}}^{\top} P E_{\mathrm{in}} \geq 0\right\}$ with basis $\left[\mathbf{x}^{\top} 1\right]^{\top}$. The change-of-basis matrix is

$$
E_{\text {in }}=\left[\begin{array}{ccccc}
I_{n_{x}} & 0 & \cdots & 0 & 0 \\
0 & 0 & \cdots & 0 & 1
\end{array}\right]
$$

Proposition 4. The quadratic inequalities (19) defined by matrices $S_{i}$ in (20) or (21), describing the candidate set $\overline{\mathcal{R}}\left(\mathcal{X}_{0}\right)$, are equivalent to the quadratic inequalities defined by matrices $M_{\text {out }}\left(S_{i}\right)=$ $E_{\text {out }}^{\top} S_{i} E_{\text {out }}$ with basis $\left[\mathbf{x}^{\top} 1\right]^{\top}$. The change-of-basis matrix is

$$
E_{\text {out }}=\left[\begin{array}{cccccc}
A_{t} & 0 & \cdots & 0 & B_{t} & c_{t} \\
0 & 0 & \cdots & 0 & 0 & 1
\end{array}\right]
$$

Proposition 5. The $Q C$ defined by $\mathcal{Q}$ in (26), satisfied by the neural network controller $\pi\left(x_{t}\right)$ in (7), is equivalent to the $Q C$ defined by $\mathcal{M}_{\text {mid }}=\left\{M_{\text {mid }}(Q) \in \mathbb{S}^{n_{x}+n_{n}+1} \mid M_{\text {mid }}(Q)=E_{\text {mid }}^{\top} Q E_{\text {mid }} \geq 0\right\}$ with basis $\left[\mathbf{x}^{\top} 1\right]^{\top}$. The change-of-basis matrix is

$$
E_{\text {mid }}=\left[\begin{array}{cc}
A & a \\
B & b \\
0 & 1
\end{array}\right] .
$$

where

$$
\begin{aligned}
A & =\left[\begin{array}{ccccccc}
W^{0} & \cdots & 0 & 0 & 0 & 0 \\
\vdots & \ddots & \vdots & \vdots & \vdots & \vdots \\
0 & \cdots & W^{\ell-1} & 0 & 0 & 0 \\
0 & \cdots & 0 & W^{\ell} & 0 & 0 \\
0 & \cdots & 0 & 0 & -I_{n_{u}} & 0
\end{array}\right] \quad a=\left[\begin{array}{c}
b^{0} \\
\vdots \\
b^{\ell-1} \\
b^{\ell}-\underline{u}_{t} \\
\bar{u}_{t}
\end{array}\right] \\
B & =\left[\begin{array}{cccccc}
0 & I_{n_{1}} & \cdots & 0 & 0 & 0 \\
\vdots & \vdots & \ddots & \vdots & \vdots & \vdots \\
0 & 0 & \cdots & I_{n_{\ell}} & 0 & 0 \\
0 & 0 & \cdots & 0 & I_{n_{u}} & 0 \\
0 & 0 & \cdots & 0 & 0 & -I_{n_{u}}
\end{array}\right] \quad b=\left[\begin{array}{c}
0 \\
\vdots \\
0 \\
-\underline{u}_{t} \\
\bar{u}_{t}
\end{array}\right] .
\end{aligned}
$$


Proof. See Appendix A.

\subsection{Over-Approximating the One-Step Reachable Set}

In the next theorem, we state our main result for over-approximating the one-step reachable set $\mathcal{R}\left(\mathcal{X}_{0}\right)$ for the closed-loop system 10 .

Theorem 1 (SDP for one-step reachable set). Consider the closed-loop system 10). Suppose the initial set $\mathcal{X}_{0}$ and the projected neural network controller $\operatorname{Proj}_{\mathcal{U}_{t}}(\pi(\cdot))$ satisfy the $Q C s$ defined by $\mathcal{M}_{\text {in }}$ and $\mathcal{M}_{\text {mid }}$, respectively, as in Proposition 3 and 5. Let $M_{\text {out }}(S)$ describe a candidate set $\overline{\mathcal{R}}\left(\mathcal{X}_{0}\right) \subseteq \mathbb{R}^{n_{x}}$ as in Proposition 4 with $S=S_{i} \in \mathbb{S}^{n_{x}+1}$. If the following LMI

$$
M_{\text {in }}(P)+M_{\text {mid }}(Q)+M_{\text {out }}(S) \preceq 0,
$$

is feasible for some matrices $(P, Q, S) \in \mathcal{P} \times \mathcal{Q} \times \mathbb{S}^{n_{x}+1}$, then $\mathcal{R}\left(\mathcal{X}_{0}\right) \subseteq \overline{\mathcal{R}}\left(\mathcal{X}_{0}\right)$.

Proof. Since the initial set $\mathcal{X}_{0}$ satisfies the QC defined by $\mathcal{M}_{\text {in }}$, we have,

$$
\left[\begin{array}{c}
\mathbf{x} \\
1
\end{array}\right]^{\top} M_{\text {in }}\left[\begin{array}{c}
\mathbf{x} \\
1
\end{array}\right] \geq 0
$$

for all $x_{0} \in \mathcal{X}_{0}$. Similarly, the projected neural network controller $\operatorname{Proj}_{\mathcal{U}_{t}}(\pi(\cdot))$ satisfying the QC defined by $\mathcal{M}_{\text {mid }}$ implies that,

$$
\left[\begin{array}{c}
\mathbf{x} \\
1
\end{array}\right]^{\top} M_{\text {mid }}\left[\begin{array}{l}
\mathbf{x} \\
1
\end{array}\right] \geq 0, \quad \forall \mathbf{x} \in \mathbb{R}^{n_{x}+n_{n}} .
$$


we have

$$
\left[\begin{array}{c}
\mathbf{x} \\
1
\end{array}\right]^{\top} M_{\text {in }}\left[\begin{array}{c}
\mathbf{x} \\
1
\end{array}\right]+\left[\begin{array}{c}
\mathbf{x} \\
1
\end{array}\right]^{\top} M_{\text {mid }}\left[\begin{array}{c}
\mathbf{x} \\
1
\end{array}\right]+\left[\begin{array}{c}
\mathbf{x} \\
1
\end{array}\right]^{\top} M_{\text {out }}\left[\begin{array}{c}
\mathbf{x} \\
1
\end{array}\right] \leq 0
$$

The first two quadratic terms in (37) are nonnegative for any $x_{0} \in \mathcal{X}_{0}$ by (35) and (36), respectively. Consequently, the last quadratic term must be nonpositive for all $x_{0} \in \mathcal{X}_{0}$,

$$
\left[\begin{array}{c}
\mathbf{x} \\
1
\end{array}\right]^{\top} M_{\text {out }}\left[\begin{array}{c}
\mathbf{x} \\
1
\end{array}\right] \leq 0
$$

By Proposition 4, the above condition is equivalent to

$$
\left[\begin{array}{l}
y \\
1
\end{array}\right]^{\top} S\left[\begin{array}{l}
y \\
1
\end{array}\right] \leq 0
$$

for all $\bar{y} \in\left\{y \mid y=f_{\pi}\left(x_{0}\right), x_{0} \in \mathcal{X}_{0}\right\}=\mathcal{R}\left(\mathcal{X}_{0}\right)$. Recall from 19 that the set of all points $y$ that satisfies (39) is the candidate set $\overline{\mathcal{R}}\left(\mathcal{X}_{0}\right)$. Therefore, we conclude that $\overline{\mathcal{R}}\left(\mathcal{X}_{0}\right)$ must be a superset of the exact one-step reachable set $\mathcal{R}\left(\mathcal{X}_{0}\right)$, i.e. $\mathcal{R}\left(\mathcal{X}_{0}\right) \subseteq \overline{\mathcal{R}}\left(\mathcal{X}_{0}\right)$. 


\subsection{Minimum-Volume Approximate Reachable Set}

Theorem 1 provides a sufficient condition for over-approximating the one-step reachable set $\mathcal{R}\left(\mathcal{X}_{0}\right)$. Now we can use this result to reformulate problem (14), which finds a minimum-volume approximate reachable set $\overline{\mathcal{R}}\left(\mathcal{X}_{0}\right)$.

If the approximate reachable set is parametrized by a polytope as in 20, , it is difficult to find a minimum-volume polytope directly. However, given a matrix $A \in \mathbb{R}^{m \times n_{x}}$ that describes the facets of the polytope, we can solve the following SDP problem,

$$
\underset{P \in \mathcal{P}, Q \in \mathcal{Q}, b_{i} \in \mathbb{R}}{\operatorname{minimize}} b_{i} \quad \text { subject to } 34 \text {, }
$$

for all $i=1, \cdots, m$. For each fixed facet $a_{i}^{\top} \in \mathbb{R}^{n_{x}}$ in $A$, SDP 40 finds the tightest halfspace $\left\{y \mid a_{i} y \leq b_{i}\right\}$ that contains $\mathcal{R}\left(\mathcal{X}_{0}\right)$. Finally, the polytopic approximate reachable set is given by the intersection of those halfsapces, i.e. $\overline{\mathcal{R}}\left(\mathcal{X}_{0}\right)=\{y \mid A y \leq b\}$, as in Section 3.2 .

If the approximate reachable set is parametrized by an ellipsoid as in (21), we can easily obtain a minimum-volume ellipsoid that encloses $\mathcal{R}\left(\mathcal{X}_{0}\right)$ by solving,

$$
\underset{\substack{P \in \mathcal{P}, Q \in \mathcal{Q}, A \in \mathbb{S}^{n}, b \in \mathbb{R}^{n_{x}}}}{\operatorname{minimize}}-\log \operatorname{det}(A) \quad \text { subject to } 34 \text {. }
$$

Note that (34) is not convex in $A$ and $b$. Nonetheless we can find a convex constraint equivalent to (34) using Schur complement. See [11] for detail.

Remark 3. In [9], an MILP-based approach is proposed for estimating forward reachable sets of dynamical systems in closed-loop with neural network controllers. If the facets are given with the same directions as the ones of the true reachable sets, then the estimated reachable sets are exact. However, this method only works for polytopic reachable sets and does not scale well with the size of the neural network, the volume, and the dimension of the initial set.

\section{Numerical Experiments}

In this section, we demonstrate our approach with two application examples. The controllers used to generate training data were implemented in YALMIP [25]. All neural network controllers were trained with ReLU activation functions and the Adam algorithm in PyTorch. We used MATLAB, CVX [15] and Mosek [1] to solve the Reach-SDP problems.

\subsection{Double Integrator}

We first consider a double integrator system

$$
x_{t+1}=\underbrace{\left[\begin{array}{ll}
1 & 1 \\
0 & 1
\end{array}\right]}_{A} x_{t}+\underbrace{\left[\begin{array}{c}
0.5 \\
1
\end{array}\right]}_{B} u_{t}
$$

discretized with sampling time $t_{s}=1$ s and subject to state and input constraints, $\mathcal{A}^{\complement}=[-5,5] \times$ $[-1,1]$ and $\mathcal{U}=[-1,1]$, respectively. We implemented a standard linear MPC following [6] with a prediction horizon $N_{\mathrm{MPC}}=10$, weighting matrices $Q=I_{2}, R=1$, the terminal region $\mathcal{O}_{\infty}^{L Q R}$ and the terminal weighting matrix $P_{\infty}$ synthesized from the discrete-time algebraic Riccati equation. The MPC is designed as a stabilizing controller which steers the system to the origin while 
satisfying the constraints. We then use the MPC to generate 2420 samples of state and input pairs $\left(x, \pi_{\mathrm{MPC}}(x)\right)$ for learning. The neural network has 2 hidden layers with 10 and 5 neurons, respectively. Our goal is to verify if all initial states in $\mathcal{X}_{0}=[2.5,3] \times[-0.25,0.25]$ can reach the set $\mathcal{G}=[-0.25,0.25] \times[-0.25,0.25]$, a region near the origin, in $N=6$ steps while avoiding $\mathcal{A}$ at all times. We computed the minimum-volume polytopic approximate reachable sets $\overline{\mathcal{R}}^{1}\left(\mathcal{X}_{0}\right), \cdots, \overline{\mathcal{R}}^{6}\left(\mathcal{X}_{0}\right)$ using the Reach-SDP introduced in Section 4 . The matrix that determines the direction of the facets is chosen as

$$
A_{\text {in }}=\left[\begin{array}{cccccccc}
1 & -1 & 0 & 0 & 1 & -1 & 1 & -1 \\
0 & 0 & 1 & -1 & -1 & 1 & 1 & -1
\end{array}\right]^{\top}
$$

As shown in Figure 2, our approach yielded a tight outer-approximation of the reachable sets and successfully verified the safety properties sought.



Figure 2: Illustration of the exact reachable sets (blue), the over-approximated reachable sets computed by Reach-SDP (red) and the goal set (green) of the double integrator. The solid black line represents the state constraint $x_{2} \geq-1$.

\section{$5.26 \mathrm{D}$ Quadrotor}

In the second example, we apply Reach-SDP to the 6D quadrotor model in [27]. In order to have a linear model as in (5), we rewrite the nonlinear quadrotor dynamics in [27] as follows,

$$
\dot{x}=\underbrace{\left[\begin{array}{cc}
0_{3 \times 3} & I_{3} \\
0_{3 \times 3} & 0_{3 \times 3}
\end{array}\right]}_{A} x+\underbrace{\left[\begin{array}{ccc}
0 & 0 & 0 \\
0 & 0 & 0 \\
0 & 0 & 0 \\
g & 0 & 0 \\
0 & -g & 0 \\
0 & 0 & 1
\end{array}\right]}_{B} \underbrace{\left[\begin{array}{c}
\tan (\theta) \\
\tan (\phi) \\
\tau
\end{array}\right]}_{u}+\underbrace{\left[\begin{array}{c}
0_{5 \times 1} \\
-g
\end{array}\right]}_{c}
$$

where $g$ is the gravitational acceleration, the state vector $x=\left[p_{x}, p_{y}, p_{z}, v_{x}, v_{y}, v_{z}\right]^{\top}$ include positions and velocities of the quadrotor in the $3 \mathrm{D}$ space and the control vector $u$ is a function of $\theta$ (pitch), $\phi$ (roll) and $\tau$ (thrust), which are the control inputs of the original model. The control task is 
to steer the quadrotor to the origin while respecting the state constraints $\mathcal{A}^{\complement}=[-5,5] \times[-5,5] \times$ $[-5,5] \times[-1,1] \times[-1,1] \times[-1,1]$ and the actuator constraints $[\theta, \phi, \tau]^{\top} \in[-\pi / 9, \pi / 9] \times[-\pi / 9, \pi / 9] \times$ $[0,2 g]$. We implemented a nonlinear MPC with a prediction horizon $N_{\mathrm{MPC}}=30$, a least-squares objective function that penalizes both states and inputs with weighting matrices $Q=I_{6}, R=I_{4}$ and the terminal constraint $x_{N_{\mathrm{MPC}}}=0$. We used the original nonlinear dynamics in [27] as the prediction model in MPC, which is discretized with a sampling time $t_{s}=0.1 \mathrm{~s}$ using the RungeKutta 4th order method. The nonlinear MPC problems were solved using SNOPT [14]. A total number of 4531 feasible samples of state and input pairs $\left(x, \pi_{\mathrm{MPC}}(x)\right)$ were generated and used to train a neural network with 2 hidden layers and 32 neurons in each layer. The initial set is given as an ellipsoid $\mathcal{X}_{0}=\mathcal{E}\left(q_{0}, Q_{0}\right)$, where $q_{0}=\left[\begin{array}{llllll}4.7 & 4.7 & 3 & 0.95 & 0 & 0\end{array}\right]^{\top}$ is the center and $Q_{0}=\operatorname{diag}\left(0.05^{2}, 0.05^{2}, 0.05^{2}, 0.01^{2}, 0.01^{2}, 0.01^{2}\right)$ is the shape matrix. Here, we want to verify if all initial states in $\mathcal{X}_{0}$ can reach the set $\mathcal{G}=[3.7,4.1] \times[2.5,3.5] \times[1.2,2.6]$, which is defined in the $\left(p_{x}, p_{y}, p_{z}\right)$-space, in $t=1$ second subject to the state and input constraints. We approximated the ellipsoidal forward reachable sets $\overline{\mathcal{R}}^{1}\left(\mathcal{X}_{0}\right), \cdots, \overline{\mathcal{R}}^{10}\left(\mathcal{X}_{0}\right)$ using the Reach-SDP. The resulting approximate reachable sets are plotted in Figure 3 and 4 which shows that our method is able to verify the given reach-avoid specifications.

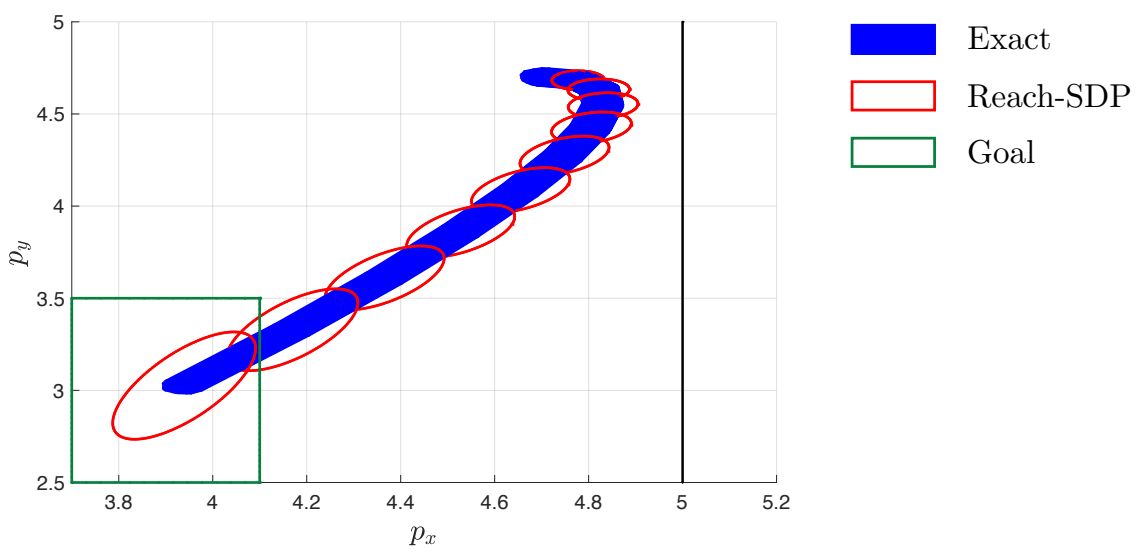

Figure 3: Illustration of the exact and approximate reachable sets of the quadrotor system in the $\left(p_{x}, p_{y}\right)$-space. The solid black line represents the state constraint $p_{x} \leq 5$.

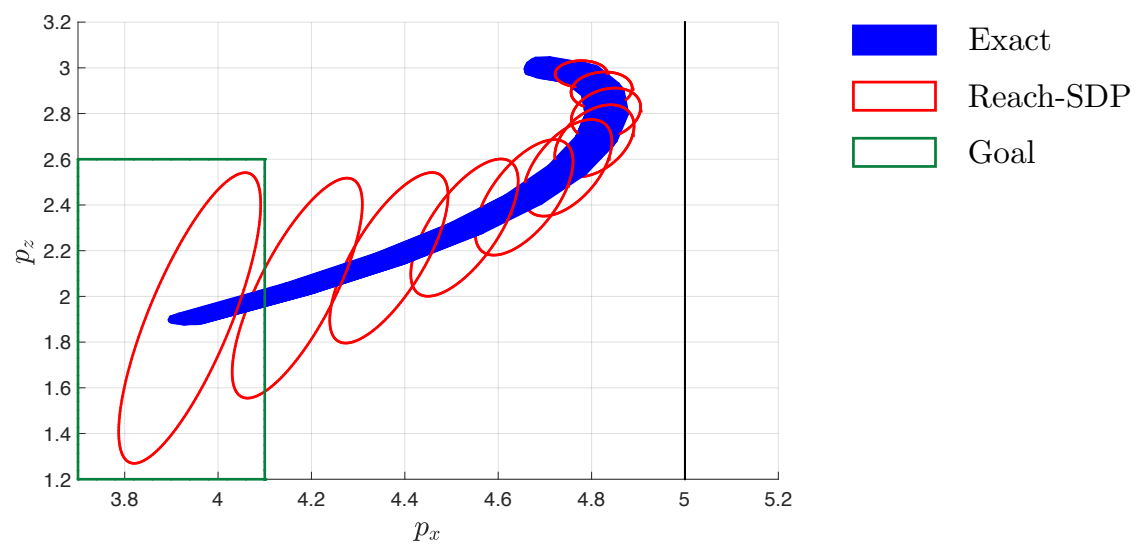

Figure 4: Illustration of the exact and approximate reachable sets of the quadrotor system in the $\left(p_{x}, p_{z}\right)$-space. 


\section{Conclusions}

In this paper, we propose the first convex-optimization-based reachability analysis method for linear systems in feedback interconnection with neural network controllers. Our approach relies on abstracting the nonlinear components of the closed-loop system by quadratic constraints. Then we show that we can compute the approximate reachable sets via semidefinite programming. Future work includes extending the current approach to incorporate nonlinear dynamics and to approximate backward reachable sets, which is useful for certifying invariance properties.

\section{A Proof of Proposition 5}

Assuming the same activation function for all neurons throughout the entire network, we can write (22) compactly as

$$
B \mathbf{x}+b=\phi(A \mathbf{x}+a)
$$

where $\mathbf{x} \in \mathbb{R}^{n_{x}+n_{n}+2 n_{u}}$ is used to define the basis vector in (28). Now, we introduce two auxiliary variables $\mathbf{y}$ and $\mathbf{z}$ such that $\mathbf{y}=A \mathbf{x}+a$ and $\mathbf{z}=B \mathbf{x}+b$. By Lemma 1, the neural network in (44) satisfies the QC defined by $\mathcal{Q}$ in (26) with basis $\left[\mathbf{y}^{\top} \mathbf{z}^{\top} 1\right]^{\top}$. Consider the congruence transformation,

$$
\left[\begin{array}{l}
\mathbf{y} \\
\mathbf{z} \\
1
\end{array}\right]=E_{\text {mid }}\left[\begin{array}{c}
\mathbf{x} \\
1
\end{array}\right] .
$$

By Lemma 2 the neural network (22) satisfies the QC defined by $\mathcal{M}_{\text {mid }}$ with basis $\left[\mathbf{x}^{\top} 1\right]^{\top}$, which concludes the proof.

\section{References}

[1] MOSEK ApS. The MOSEK optimization toolbox for MATLAB manual. Version 8.1., 2017.

[2] Alberto Bemporad and Manfred Morari. Verification of hybrid systems via mathematical programming. In International Workshop on Hybrid Systems: Computation and Control, pages 31-45. Springer, 1999.

[3] Alberto Bemporad, Fabio Danilo Torrisi, and Manfred Morari. Optimization-based verification and stability characterization of piecewise affine and hybrid systems. In International Workshop on Hybrid Systems: Computation and Control, pages 45-58. Springer, 2000.

[4] Franco Blanchini and Stefano Miani. Set-theoretic methods in control. Springer, 2008.

[5] Mariusz Bojarski, Davide Del Testa, Daniel Dworakowski, Bernhard Firner, Beat Flepp, Prasoon Goyal, Lawrence D Jackel, Mathew Monfort, Urs Muller, Jiakai Zhang, et al. End to end learning for self-driving cars. arXiv preprint arXiv:1604.07316, 2016.

[6] Francesco Borrelli, Alberto Bemporad, and Manfred Morari. Predictive control for linear and hybrid systems. Cambridge University Press, 2017.

[7] Steven Chen, Kelsey Saulnier, Nikolay Atanasov, Daniel D Lee, Vijay Kumar, George J Pappas, and Manfred Morari. Approximating explicit model predictive control using constrained neural networks. In 2018 Annual American control conference (ACC), pages 1520-1527. IEEE, 2018. 
[8] Souradeep Dutta, Xin Chen, and Sriram Sankaranarayanan. Reachability analysis for neural feedback systems using regressive polynomial rule inference. In Proceedings of the 22nd ACM International Conference on Hybrid Systems: Computation and Control, pages 157-168, 2019.

[9] Souradeep Dutta, Susmit Jha, Sriram Sanakaranarayanan, and Ashish Tiwari. Output range analysis for deep neural networks. arXiv preprint arXiv:1709.09130, 2017.

[10] Ruediger Ehlers. Formal verification of piece-wise linear feed-forward neural networks. In International Symposium on Automated Technology for Verification and Analysis, pages 269286. Springer, 2017.

[11] Mahyar Fazlyab, Manfred Morari, and George J Pappas. Probabilistic verification and reachability analysis of neural networks via semidefinite programming. arXiv preprint arXiv:1910.04249, 2019.

[12] Mahyar Fazlyab, Manfred Morari, and George J Pappas. Safety verification and robustness analysis of neural networks via quadratic constraints and semidefinite programming. arXiv preprint arXiv:1903.01287, 2019.

[13] Mahyar Fazlyab, Alexander Robey, Hamed Hassani, Manfred Morari, and George Pappas. Efficient and accurate estimation of lipschitz constants for deep neural networks. In Advances in Neural Information Processing Systems, pages 11423-11434, 2019.

[14] Philip E. Gill, Walter Murray, and Michael A. Saunders. SNOPT: An SQP algorithm for large-scale constrained optimization. SIAM Rev., 47:99-131, 2005.

[15] Michael Grant, Stephen Boyd, and Yinyu Ye. Cvx: Matlab software for disciplined convex programming, 2009.

[16] M. Herceg, M. Kvasnica, C.N. Jones, and M. Morari. Multi-Parametric Toolbox 3.0. In Proc. of the European Control Conference, pages 502-510, Zürich, Switzerland, July 17-19 2013.

[17] Michael Hertneck, Johannes Köhler, Sebastian Trimpe, and Frank Allgöwer. Learning an approximate model predictive controller with guarantees. IEEE Control Systems Letters, $2(3): 543-548,2018$.

[18] Chao Huang, Jiameng Fan, Wenchao Li, Xin Chen, and Qi Zhu. Reachnn: Reachability analysis of neural-network controlled systems. ACM Transactions on Embedded Computing Systems (TECS), 18(5s):1-22, 2019.

[19] Xiaowei Huang, Marta Kwiatkowska, Sen Wang, and Min Wu. Safety verification of deep neural networks. In International Conference on Computer Aided Verification, pages 3-29. Springer, 2017.

[20] Radoslav Ivanov, James Weimer, Rajeev Alur, George J Pappas, and Insup Lee. Verisig: verifying safety properties of hybrid systems with neural network controllers. In Proceedings of the 22nd ACM International Conference on Hybrid Systems: Computation and Control, pages 169-178, 2019.

[21] Guy Katz, Clark Barrett, David L Dill, Kyle Julian, and Mykel J Kochenderfer. Reluplex: An efficient smt solver for verifying deep neural networks. In International Conference on Computer Aided Verification, pages 97-117. Springer, 2017. 
[22] J Zico Kolter and Eric Wong. Provable defenses against adversarial examples via the convex outer adversarial polytope. arXiv preprint arXiv:1711.00851, 1(2):3, 2017.

[23] Alexey Kurakin, Ian Goodfellow, and Samy Bengio. Adversarial examples in the physical world. arXiv preprint arXiv:1607.02533, 2016.

[24] Robert P Kurshan. Computer-aided verification of coordinating processes: the automatatheoretic approach. Princeton university press, 2014.

[25] Johan Lofberg. Yalmip: A toolbox for modeling and optimization in matlab. In 2004 IEEE international conference on robotics and automation (IEEE Cat. No. 04CH37508), pages 284289. IEEE, 2004.

[26] Alessio Lomuscio and Lalit Maganti. An approach to reachability analysis for feed-forward relu neural networks. arXiv preprint arXiv:1706.07351, 2017.

[27] Diego Manzanas Lopez, Patrick Musau, Hoang-Dung Tran, and Taylor T. Johnson. Verification of closed-loop systems with neural network controllers. In Goran Frehse and Matthias Althoff, editors, ARCH19. 6th International Workshop on Applied Verification of Continuous and Hybrid Systems, volume 61 of EPiC Series in Computing, pages 201-210. EasyChair, 2019.

[28] Yunpeng Pan, Ching-An Cheng, Kamil Saigol, Keuntaek Lee, Xinyan Yan, Evangelos Theodorou, and Byron Boots. Agile autonomous driving using end-to-end deep imitation learning. arXiv preprint arXiv:1709.07174, 2017.

[29] Nicolas Papernot, Patrick McDaniel, Somesh Jha, Matt Fredrikson, Z Berkay Celik, and Ananthram Swami. The limitations of deep learning in adversarial settings. In Security and Privacy (EuroSEP), 2016 IEEE European Symposium on, pages 372-387. IEEE, 2016.

[30] Stephen Prajna and Ali Jadbabaie. Safety verification of hybrid systems using barrier certificates. In International Workshop on Hybrid Systems: Computation and Control, pages 477-492. Springer, 2004.

[31] Ahmed H Qureshi, Anthony Simeonov, Mayur J Bency, and Michael C Yip. Motion planning networks. In 2019 International Conference on Robotics and Automation (ICRA), pages 21182124. IEEE, 2019.

[32] Aditi Raghunathan, Jacob Steinhardt, and Percy S Liang. Semidefinite relaxations for certifying robustness to adversarial examples. In Advances in Neural Information Processing Systems, pages 10900-10910, 2018.

[33] Claire J Tomlin, Ian Mitchell, Alexandre M Bayen, and Meeko Oishi. Computational techniques for the verification of hybrid systems. Proceedings of the IEEE, 91(7):986-1001, 2003.

[34] Min Wen and Ufuk Topcu. Constrained cross-entropy method for safe reinforcement learning. In Advances in Neural Information Processing Systems, pages 7450-7460, 2018.

[35] Tianhao Zhang, Gregory Kahn, Sergey Levine, and Pieter Abbeel. Learning deep control policies for autonomous aerial vehicles with mpc-guided policy search. In 2016 IEEE international conference on robotics and automation (ICRA), pages 528-535. IEEE, 2016. 